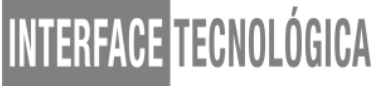

\section{ESTUDO DA APLICAÇÃO SAP HANA EM BANCO DE DADOS}

\section{STUDY OF THE SAP HANA APPLICATION IN DATABASE}

\author{
Natalia Barbizan Fernandes - nbarbizanfernandes@gmail.com \\ Eder Carlos Salazar Sotto - eder.sotto@ fatectq.edu.br \\ Faculdade de Tecnologia de Taquaritinga (FATEC) - SP - Brasil
}

DOI: 10.31510/infa.v15i1.338

\section{RESUMO}

Sistemas de informação são fundamentais para o mundo dos negócios, de pequeno a grande porte, permitindo inclusive a automatização de processos na abstração dos dados. Para gerar uma informação precisa em tempo reduzido, um dos principais componentes é a base de dados, encarregada do armazenamento de dados vindos de várias fontes distintas, permitindo posterior extração, o que agrega valor em uma tomada decisão da organização. Este artigo tem como objetivo estudar uma aplicação SAP HANA, sendo esta a conjunção de uma plataforma de software e hardware com uma única base de dados in-memory. Foi utilizada como metodologia a pesquisa bibliográfica.

Palavras-chaves: Base Dados. Informação. Sistemas. SAP HANA.

\begin{abstract}
Information systems are fundamental to the business world, from small to large, allowing even the automation of processes in the abstraction of data. To generate accurate information in a reduced time, one of the main components is the database, in charge of storing data coming from several different sources to allow later extraction, which adds value in a decision making of the organization. This article aims to study a SAP HANA application, which is the conjunction of a software and hardware platform with a single in-memory database. The research methodology used is through bibliographic research.
\end{abstract}

Keywords: Database. Information. Systems. SAP HANA.

\section{INTRODUÇÃO}

O uso da tecnologia da informação no meio empresarial deve ser pautado da otimização do uso dos recursos computacionais, para que os investimentos nestes recursos sejam feitos de acordo com a real necessidade (NICODEMOS, 2012). 
O banco de dados é de suma importância para o funcionamento de qualquer sistema de informação. Quando for ao banco retirar dinheiro ou realizar um deposito bancário, ou o simples ato de adquirir produtos em um supermercado, está indiretamente alimentando o banco de dados de algum sistema de informação (ELMASRI e NAVATHE, 2005).

Segundo Meira (2013), um banco de dados é uma coleção de dados para determinado fim, de forma que estes dados se relacionem e criem um sentido. Os bancos de dados são de vital importância e são considerados o principal componente dos sistemas de informação. No entanto, para se acessar os dados de um banco de dados, os sistemas de informação utilizam um Sistema Gerenciador de Banco de Dados (SGBD), que é uma coleção de programas que permitem a construção de bancos de dados e a manipulação de dados.

O objetivo deste artigo é apresentar a ferramenta analítica de alto desempenho SAP High Performance Analytic Appliance (SAP HANA) sob operações de negócios de uma empresa utilizando grandes volumes de informações, retornando em tempo real informações para melhores decisões da organização a partir de qualquer fonte de dados. Este trabalho também abordará sobre as vantagens de uso desta aplicação, bem como da transição para esta plataforma de banco de dados.

O SAP HANA passou por imensos investimentos, passando de um banco de dados a uma plataforma ideal para auxiliar empresas para economia digital, com a tecnologia inmemory, e está sendo considerado um investimento promissor para uma nova geração de infraestrutura de TI. A metodologia utilizada para o desenvolvimento deste estudo será a pesquisa bibliográfica (SAP, 2017).

O estudo foi estruturado iniciando-se pelo primeiro Capítulo com a Introdução, seguindo com o Capítulo 2 com um breve histórico sobre bancos de dados relacionais. $\mathrm{O}$ Capítulo 3 apresenta o conceito do SAP HANA, seu funcionamento, recursos e benefícios. No Capítulo 4, apresentam-se as conclusões do estudo, e por fim as referências utilizadas.

\section{BANCO DE DADOS}

Nos dias atuais, a informação é considerada o principal ativo de comunicação pela sociedade, sendo verbal, escrita ou por meio de aplicações que as armazenam para no futuro realizar sua reprodução. Sendo assim, será apresentado um breve histórico do banco de dados, mostrando sua evolução e seu tempo cronológico. 
Na década de 1950, com o início do uso dos computadores para fins comerciais, o armazenamento em fitas magnéticas e cartões perfurados rapidamente se tornou obsoleto. Entre 1960 e 1970, inicializava-se o uso dos bancos de dados de modelo hierárquico, do qual é apresentado um exemplo na Figura 1.

Figura 1 - Exemplo de modelo de dados hierárquico
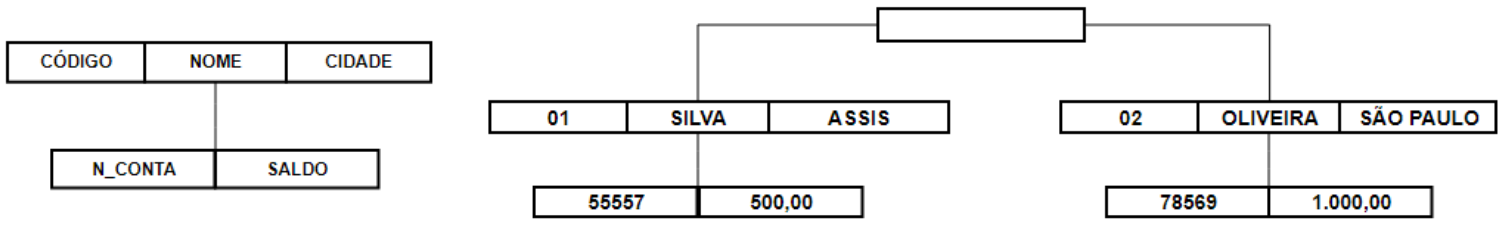

Fonte: Elaborada pelos autores (2018)

O modelo de dados hierárquico surgiu como um paradigma diante do crescimento da capacidade de armazenamento e o surgimento dos primeiros discos rígidos magnéticos. Seu meio de acesso era feito de modo sequencial e percorrendo-se o modelo de árvore, indo do topo até a folha da esquerda para a direita, onde estava armazenado o registro procurado. $\mathrm{O}$ relacionamento era feito através de link, sendo que estes eram manipulados um de cada vez, e sua consulta era, um tanto quanto, demorada e complexa (PEREIRA, 2013).

Posteriormente, por meio de Charles Bachman que foi especialista em banco de dados, reconhecido em 1973 pelo prêmio de Turing por sua contribuição fundamental à tecnologia de banco de dados, surgiu os bancos de dados em rede, os quais são representados por coleções de registros que possuem associações, eliminando todo o conceito hierárquico apresentado pelo modelo anterior. O método de acessar os dados está ligado diretamente a seu relacionamento e suas consultas, continuando a serem complexas, podendo percorrer os registros com os links em qualquer sentido até chegar a seu destino. Seu exemplo de estrutura está representado na Figura 2 por caixas e linhas (PEREIRA, 2013). 
Figura 2 - Exemplo de modelo de dados em rede

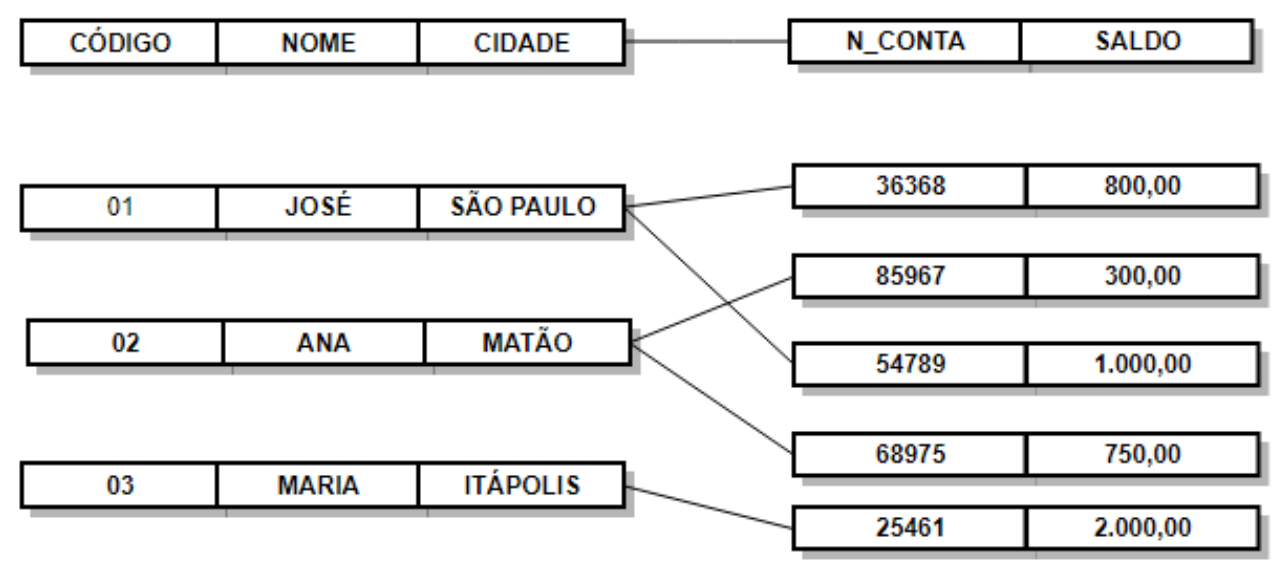

Fonte: Elaborada pelos autores (2018)

Em 1970, Edgar Franck Codd foi um matemático que desenvolveu o modelo de banco de dados relacional, quando era pesquisador no laboratório da IBM. Propõe o modelo relacional, de modo que dados são armazenados em tabelas como mostra a Figura 3. Este paradigma se tornou referência e é muito utilizado até hoje pela grande maioria dos Sistemas Gerenciadores de Banco de Dados (PEREIRA, 2013).

Figura 3 - Exemplo de instância de uma tabela do modelo de dados relacional

\begin{tabular}{|c|c|c|}
\hline CÓDIGO & NOME & CIDADE \\
\hline 01 & LUCAS & TAQUARITINGA \\
\hline 02 & MARIANA & BEBEDOURO \\
\hline 03 & FABRICIO & CATANDUVA \\
\hline & & \\
\hline
\end{tabular}

Fonte: Elaborada pelos autores (2018)

Segundo PEREIRA (2013), em meados 1980 houve o surgimento dos bancos de dados orientados a objetos (BDOO), e seu principal objetivo foi criar um banco de dados que suportasse aplicações de objetos complexos, como textos, imagens e gráficos, como é apresentado na Figura 4. 
Figura 4 - Exemplo de modelo de dados orientados a objetos

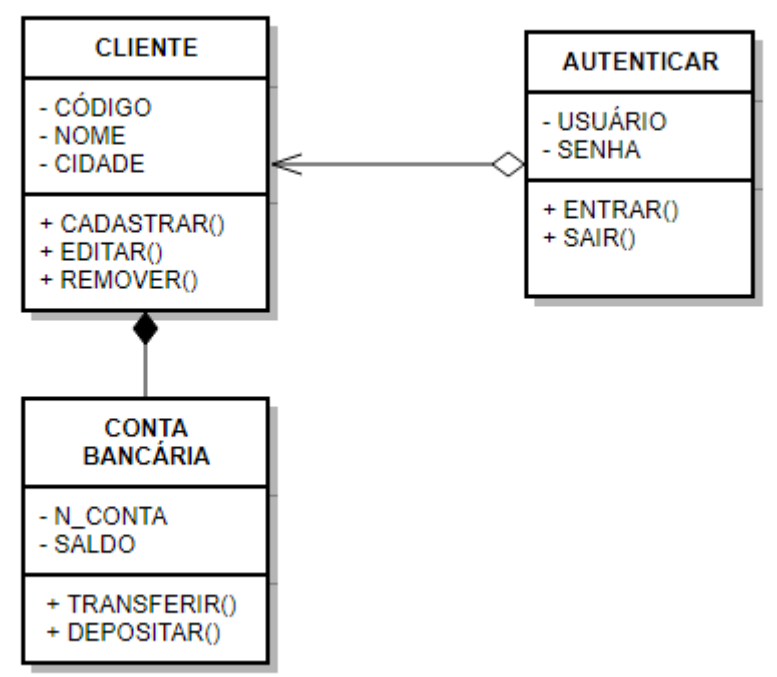

Fonte: Elaborada pelos autores (2018)

Os Bancos de Dados Objeto Relacionais (BDOR) surgiram por volta de 1990, pelo fato da grande troca de dados que já estavam sendo transmitidos na web diante do início do comércio eletrônico (E-commerce). A world wide web estava interligada por uma vasta rede de computadores e vinha impulsionando a internet a ficar disponível vinte quatro horas por dia. $\mathrm{O}$ BDOR representado na Figura 5 mescla o conceito de banco de dados relacional (BDR) com o conceito de banco de dados orientado a objetos (BDOO), unindo recursos e vantagens do uso que cada um dos paradigmas (ELMASRI e NAVATHE, 2005).

Figura 5 - Exemplo de modelo de dados objetos relacionais

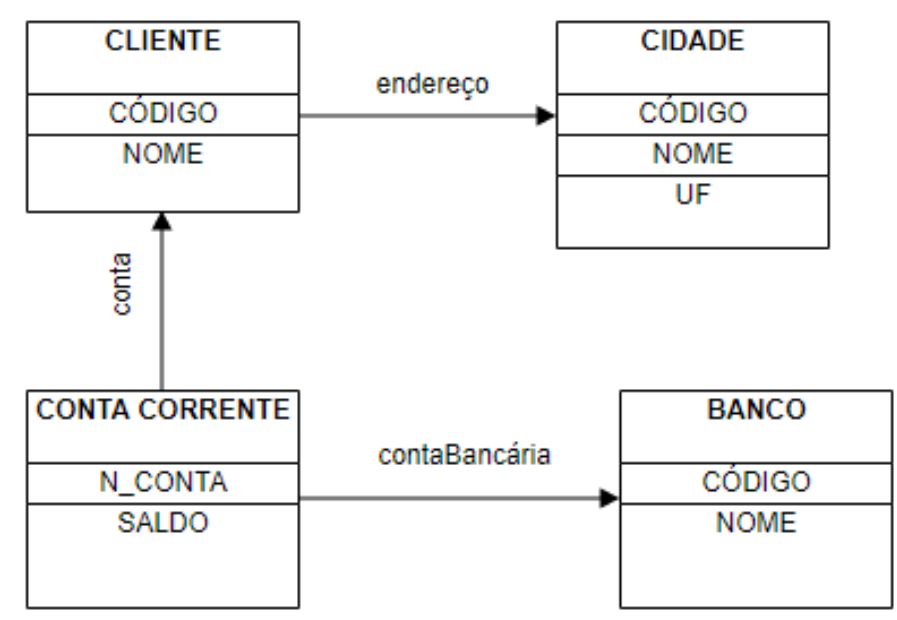

Fonte: Elaborada pelos autores (2018) 


\section{WTEREHAESTEENOLOGGCA}

Nos anos 2000 inicia-se a geração dos SGBD's avançados, momento em que os já tradicionais bancos de dados relacionais estão consolidados, obrigando pesquisadores desta área a inovar nas funcionalidades em meio aos meios de armazenamento em evolução, para serem capazes de suportar as novas aplicações que estavam surgindo no mundo da tecnologia da informação relacionados a:

- Armazenamento Móvel e pessoal: refere-se ao grande número de dispositivos pessoais móveis. O armazenamento dos dados é feito em cada dispositivo móvel pessoal, tornando-o proprietário administrador de seu banco de dados;

- Armazenamento Multimídia: é o armazenamento específico de sons, vídeos e imagens;

- Armazenamento Espacial e Geográfico: é o armazenamento das informações espaciais sobre tempo ou os mapas usados em sistemas geográficos (PEREIRA, 2013).

\section{APLICAÇÃO DE BANCO DE DADOS SAP HANA}

O SAP HANA High Performance Analytic Appliance (ferramenta analítica de alto desempenho) é uma aplicação de software em conjunto com hardware, que em 2011 foi apresentado como base de dados pela empresa SAP.

O SAP HANA surgiu com o intuito de permitir a criação de aplicativos analíticos que necessitavam de alta velocidade de processamento e acesso aos dados. Com a tecnologia inmemory, o banco de dados SAP HANA mantêm os dados recebidos na memória de trabalho, ou seja, na memória RAM, fazendo uma análise produtiva. Segundo SAP Notícias Brasil (2015), o SAP HANA transforma-se em 2013 em uma plataforma, utilizando os algoritmos de programação avançados para aplicações empresariais (ABAP advanced business application programming). ABAP é uma linguagem de programação de alto nível, desenvolvida pela empresa de software SAP. Sua funcionalidade é compatível com os requisitos pela atomicidade, consistência, isolamento e padrões de durabilidade ACID (SAP, 2017). 


\subsection{Como o SAP HANA funciona}

O HANA é um banco de dados e uma plataforma. Toda leitura e escrita é realizada em memória, e não feita como nos bancos de dados tradicionais que buscam dados armazenados em disco e após isso realizam a leitura e escrita. Logicamente o SAP HANA possui disco para armazenamento, onde os dados são gravados após todo o processamento analítico (COSTA, 2017).

Para COSTA (2017), seu funcionamento básico e diferencial do SAP HANA é devido ao uso da RAM. Enquanto nas bases robustas tradicionais, o uso é baseado em discos. Na base de dados em memória, o paralelismo irá utilizar recursos computacionais através do modo em que o conjunto de dados será particionado individualmente.

A principal característica desta tecnologia é que os dados são guardados em memória, o que diminui consideravelmente o tempo de acesso, um exemplo de hierarquia de memória pode ser visto na Figura 6, onde é evidente a diferença em tempos de acesso a disco (memória persistente) e a memória principal (memória volátil).

Figura 6 - Hierarquia de memória em disco e RAM

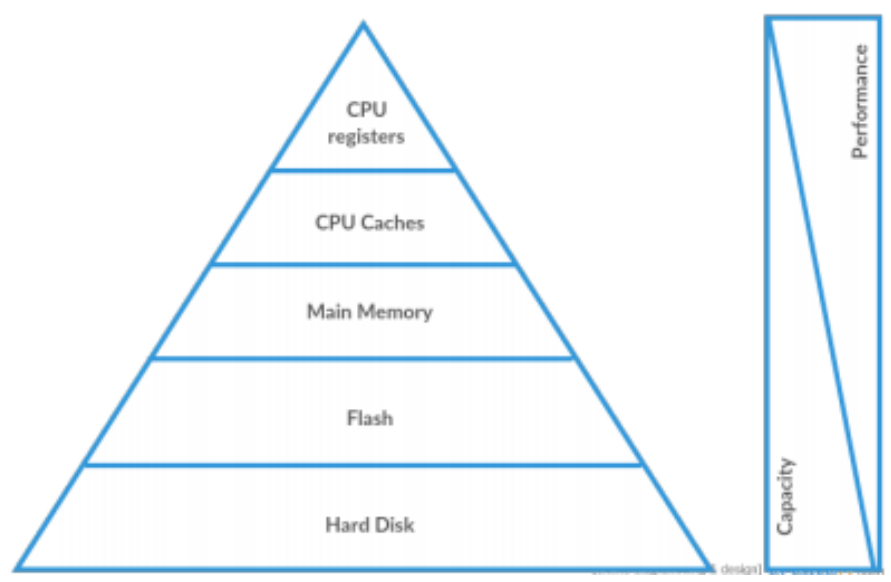

Fonte: Borges, 2016, p. 11

Estes sistemas mantêm uma cópia primária dos seus dados em memória de acesso aleatório RAM, enquanto que o disco apenas continua a ser usado para efeitos de persistência, backup e recuperação da informação (BORGES, 2016). 


\subsection{Tecnologia SAP HANA}

O SAP HANA armazena os dados em formato colunar ou em linha, mas seu foco é o formato colunar, que foi feito para suportar transações e processamento analítico em mesma representação de dados. O idioma da interface é a linguagem de consulta estruturada (SQL) e as propriedades de atomicidade, consistência, isolamento e durabilidade (ACID) concedem ao gestor de transações a possibilidade de coordenar as transações da base de dados, controlar o isolamento transacional e rastrear as transações em execução e terminadas. (LEUKERT e PLATTNER, 2015).

Seu sistema possui componentes de conectar e administrar, uma vez estabelecida a conexão ele apresentará as seguintes funções: linguagem de consulta estruturada (SQL), conjunto de instruções em SQL na função de criar, editar, exibir, executar e excluir arquivos de script (SQL Script), a consulta básica de expressões multidimensionais (MDX) com instrução select retornando de um subconjunto de dados multidimensionais, a funcionalidade processamento de gráficos, que permite a execução de operações gráficas a partir todos os dados armazenados (WIPE Graphs) ou funções personalizadas de planejamento (FOX).

O princípio de controle de concorrência de multiversão (MVCC) permite a leitura de gravações de longa execução sem bloquear transações de atualização e, juntamente com timetravel, possibilita executar comandos temporais dentro do motor relacional. As linguagens suportadas são traduzidas para compilar nas camadas internas específicas do modelo de cálculo e a execução destes modelos fica a cargo do motor de cálculo. O armazenamento em memória possui três motores: motor relacional para suportar representações físicas orientadas a linha e colunas de tabelas relacionais. O motor de grafo suporta processamento de grafos de dados com uma escrita flexível, nas aplicações de recursos de enormes números e interdependência complexa. Já, o motor texto, fornece capacidade de indexação e pesquisas de texto, com fuzzy search, capaz de tolerar erros de escrita e pesquisa baseadas em regras linguísticas. O exemplo da arquitetura é apresentado na Figura 7 (COSTA, 2017). 
Figura 7 - Arquitetura da base de dados SAP HANA

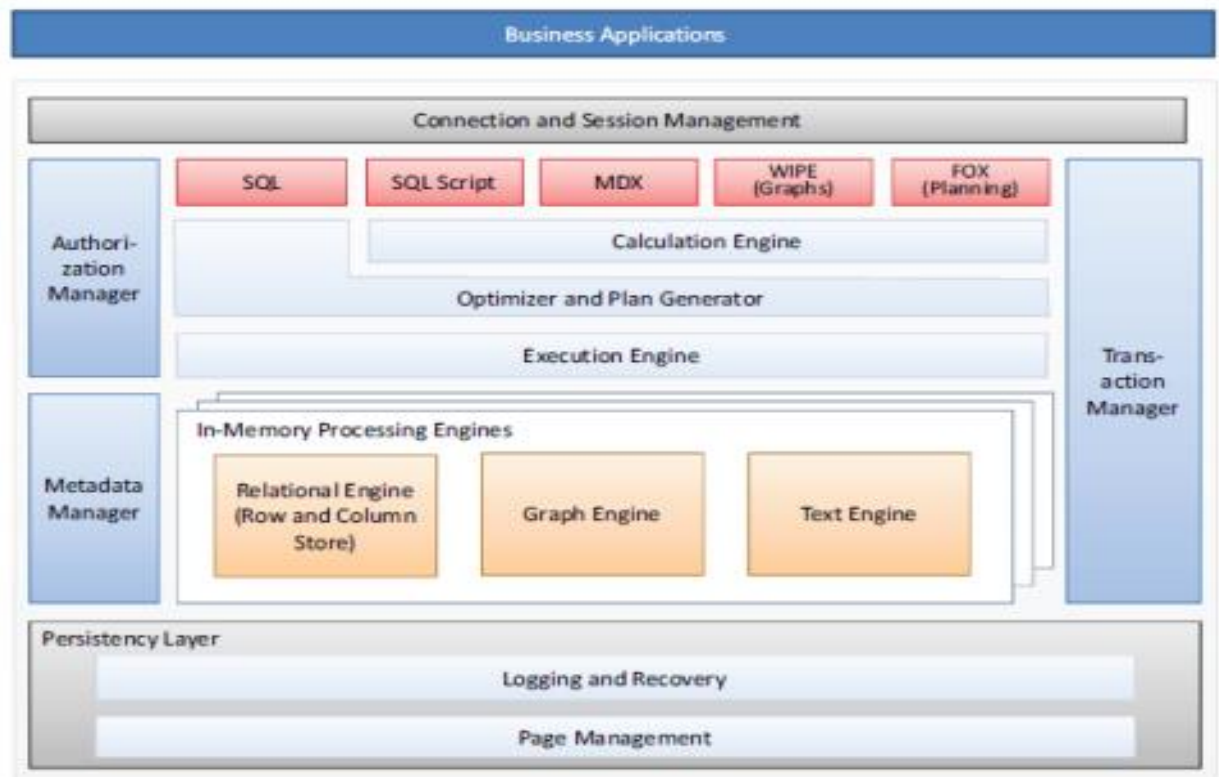

Fonte: Costa, 2017, p.18

\subsection{Recursos e benefícios do SAP HANA}

Segundo a SAP (2017), a seguir são apresentados alguns recursos e benefícios oferecidos pela aplicação SAP HANA:

Serviços de Banco de Dados: No SAP HANA há um sistema gerenciador de banco de dados relacional, do inglês relational database management system (RDBMS). Ele em conjunto com o processamento analítico online (OLAP) e processamento de transações em tempo real (OLTP) em um único banco de dados in-memory. Esse banco de dados in-memory colunar é compatível com as regras de atomicidade, consistência, isolamento e durabilidade (ACID). Os dados são armazenados compactados, fazendo processamento paralelo nos núcleos do multiprocessador, suportando o comando de múltiplos dados com instrução única (SIMD).

Processamento de Funções Analíticas:

- Processamento de Dados Espaciais: Mecanismo espacial de multicamadas. Oferece suporte a colunas espaciais, métodos de acesso espacial e sistemas de referência espacial com único banco de dados para seus dados espaciais e de negócios; 
- $\quad$ Funções Analíticas Preditivas e Processamento: Prever resultados futuros para melhor tomada decisão com precisão do Big Data, analisando qualquer tipo de dados;

- $\quad$ Processamento de Dados em série: Processamento e monitoração dos dados empresariais para avaliar impactos no negócio, como monitoração de preço, padrões sazonais, eficiência do computador em consumo de energia e rede;

- $\quad$ Processamento de Streaming de Dados: Com a função de analisar e processar dados empresariais, comparando-os as informações que chegam de várias fontes, identificando ameaças e oportunidades emergentes, para tomar providências para se regularizar;

- $\quad$ Processamento de Dados gráficos: Utilizar ferramentas de modelagem e visualização para criar e navegar em gráficos, calculando caminhos mais curtos e entendendo os relacionamentos através da obtenção de dados do negócio;

Desenvolvimento de Aplicativos: A Plataforma SAP HANA oferece uma ampla gama de ferramentas para desenvolvimento de aplicativos com serviços de desenvolvimento, ferramentas, linguagens, biblioteca de funções e gestão do clico de vida do aplicativo.

Acesso a dados: Obtém fonte de dados sem influenciar no desempenho da plataforma com serviços de federação de dados, disponibilizando a consulta dos dados remotos através da sincronização bidirecional de dados, tanto no SAP HANA como em outros bancos incorporados e localizados na rede. Tudo isso em conjunto da integração e replicação de dados, qualidade e confiabilidade dos dados e sincronização remota de dados.

Administração: As atualizações não afetam o desempenho da produção dos demais setores, garantindo a continuidade dos negócios em caso na recuperação de desastres. Utilizam virtualizações nas instalações e implementações de produção e operação.

Segurança: Gerenciamento de acesso seguro as informações com a gestão de funções, privilégios, usuários e identidades. Registro de auditoria e autenticação.

\subsection{Investimentos e o tempo envolvido na transição para desenvolvimento da própria plataforma de banco de dados}

Houve uma mudança muito complexa em relação a todo o investimento, tempo, projetos, pessoas e custos envolvidos no desenvolvimento do SAP HANA. A SAP anteriormente dependia da Oracle em todo software de armazenamento de dados. Contudo, todo 
software adquirido por um cliente da SAP era basicamente obrigado a adquirir um software de banco de dados para armazenar os dados, pois uma empresa era aliada a outra no mercado de negócios de TI. Com isso, os clientes adquiriam também o SGBD da Oracle para funcionamento completo do sistema. Isso fazia com que o cliente assumisse duas licenças, sendo uma da Oracle e outa da SAP. O SAP HANA foi desenvolvido para oferecer um software completo junto a seu próprio banco de dados, permitindo ao cliente investimento único e uma única licença de software com a SAP (LOBO, 2013).

\section{CONCLUSÃO}

O banco de dados é um componente essencial em qualquer sistema de informação, tendo a função de armazenar dados extraídos de várias fontes. As empresas precisam ter seus dados armazenados em um banco de dados que permita acesso ágil a essas informações, as quais fornecem recursos cruciais para a tomada de decisão e competitividade dessas empresas.

O SAP HANA permite a criação de aplicações ágeis, seguindo todos os padrões e normas de acesso aos dados, além de ser uma solução segura. Possibilita o processamento de grande volume de dados, permitindo analisar dados empresariais e avaliar impactos no negócio. A implantação requer o envolvimento de todos os processos organizacionais bem como o treinamento de pessoal.

A partir dos estudos realizados é possível concluir que é uma aplicação completa e sem dúvida nenhuma um diferencial de grande importância para a empresa que o utiliza, e que, se utilizada de forma adequada, possibilitará obter resultados concretos e de suma importância para a tomada de decisão nos negócios.

\section{REFERÊNCIAS}

BORGES, G. B. M. M. Study of SAP HANA in the in-memory context. Faculdade de Engenharia da Universidade do Porto, 27 jun. 2016. Disponível em: < https://repositorioaberto.up.pt/bitstream/10216/85310/2/142481.pdf >. Acesso em: 22 fev. 2018

COSTA, M.M.L.R. Estudo de dados industriais no contexto de Mobility SAP HANA. Faculdade de Engenharia da Universidade do Porto, 5 set. 2017. Disponível em: < https://repositorio-aberto.up.pt/bitstream/10216/106811/2/207622.pdf>. Acesso em: 22 fev. 2018. 
ELMASRI, R.; NAVATHE, S. Sistemas de Banco de Dados. $4^{\circ}$ ed. Tradução de M. G. Pinheiro et al. Revisão de Técnica de L. R. Figueiredo. São Paulo: Pearson Education, 2005. 798 p.

LEUKERT, B; PLATTNER, H. The in-memory revolution. How SAP HANA enable business of the future. United States. Springer, 22 Jun. 2015. 275 p. Disponível em: < https://books.google.com.br/books?id=6IdNCwAAQBAJ\&printsec=frontcover\&hl=pt$\mathrm{BR} \# \mathrm{v}=$ onepage $\& \mathrm{q} \& \mathrm{f}=$ false $>$. Acesso em: 20 nov. 2017.

LOBO, A. P. SAP declara guerra contra Oracle e a IBM no Brasil. Convergência Digital, 23 jan. 2013. Disponível em: <http://www.convergenciadigital.com.br/cgi/cgilua.exe/sys/start.htm?UserActiveTemplate=si te\&infoid=32874\&sid=5>. Acesso em: 21 fev. 2018.

MEIRA, R. Banco de Dados. IFBA - Campus Ilhéus, 2013. Disponível em: < http://www.regilan.com.br/wp-content/uploads/2013/10/Apostila-Banco-de-Dados.pdf>.

Acesso em: 14 set. 2017.

NICODEMOS, R. B. Introdução tecnologia da informação. Portal Educação, Campo Grande, 28 nov. 2012. Disponível em: <https://www.portaleducacao.com.br/conteudo/artigos/educacao-fisica/introducao-atecnologia-da-informacao/22319>. Acesso em: 02 out. 2017.

PEREIRA, A. A História do Banco de Dados, 2013. Disponível em: <https://prezi.com/cgdqpfeipvvy/a-historia-do-banco-de-dados/>. Acesso em: 30 out. 2017.

SAP, N. B. Mas o que é SAP HANA? , 09 set. 2015. Disponível em: < https://news.sap.com/brazil/2015/09/09/mas-o-que-e-sap-hana/>. Acesso em: 20 nov. 2017.

SAP. Como SAP HANA funciona? , 2017. Disponível em: < https://www.sap.com/brazil/products/hana.html\#>. Acesso em: 22 nov. 17.

SAP. Processamento de funções analíticas: Obtenha novos insights do processamento avançado de funções analíticas no SAP HANA, 2017. Disponível em: < https://www.sap.com/brazil/products/hana/features/advanced-analytics.html >. Acesso em: 29 dez. 2017.

SAP. Síntese do SAP HANA: Recursos e Benefícios, 2017. Disponível em: < https://www.sap.com/brazil/products/hana/features.html >. Acesso em: 29 dez. 2017. 